\title{
Conversation-based Learning Agents Framework
}

\author{
Choo-Peng Tan, Choo-Kim Tan, Siong-Hoe Lau, Shaun Ng Wes \\ Faculty of Information Science and Technology, Multimedia University, 75450 Melaka, Malaysia \\ Correspondence Author: Choo-Peng Tan, Faculty of Information Science and Technology, Multimedia University, 75450 Melaka, Malaysia. \\ E-mail:- cptan@mmu.edu.my.
}

Received date: 15 August 2019, Accepted date: 12 October 2019, Online date: 28 October 2019

Copyright: $\odot 2019$ Choo-Peng Tan et al, This is an open-access article distributed under the terms of the Creative Commons Attribution License, which permits unrestricted use, distribution, and reproduction in any medium, provided the original author and source are credited.

\begin{abstract}
Student's performance and achievement is always a concern. Teaching and learning process with not only blended learning but with learning agents too is found benefited. In Malaysia, it was found that incorporating conversationbased agents in teaching and learning is very rarely. Thus, this project aims to propose a framework to develop a conversation-based agent that assists students' learning in blended learning and to examine the effects of incorporating conversation-based agent on students' achievement in mathematics. Results from phase I showed that all students had never used the conversation-based learning agents in their learning and they are looking forward to use the conversation-based learning agents which they believe that the agents will make the learning fun and interesting. It is hoped that the framework that will be designed and developed in this study is significant to the instructional designers and educators in helping students to reduce difficulties and anxiety level in mathematics and thus improve performance in mathematics.
\end{abstract}

Keywords: conversation-based agents; mathematics achievement

\section{INTRODUCTION}

It has been in the last century, the famous mathematician Poincare (1908/52) described impressively on students' difficulties to understand mathematical ideas (Rösken \& Rolka, 2007). Hence, we could see in the various nations, in general, that students' achievement in mathematics is unsatisfactory and they have negative attitude towards mathematics (McCraty et al, 2000). A study done by Ma (2012) showed that "the feeling of enjoyment, not the feeling of difficulty, directly affected mathematics achievement." According to Zan, R. and Di Martino, P., (2007), "when it refers to emotion, 'positive' normally means 'perceived as pleasurable'. So, anxiety when confronting a problem is seen as 'negative', while pleasure in doing mathematics is evaluated as 'positive'." Thus, creating an interactive and enjoyable learning process is another concern that educators should consider. In order to achieve successful learning in mathematics, students should be given the opportunity to communicate mathematically, reasoning mathematically, develop self-confidence to solve problem (Johnson and Johnson, 1990). Due to the rapid growth of information technology, it has to transform all the aspects of our life including teaching and learning of mathematics from the traditional way of using technology tools. Besides that, the World Wide Web (WWW) has rapidly and drastically become an important and powerful medium not only for communication but for education, research, commerce and many other fields since it was first introduced in 1990s. With its rapid growth, it has created a vast set of resources that can be accessed from anywhere and at any time. The creation of this vast virtual information repository provides a very good e-learning environment.

Various teaching practices, approaches and/ or strategies, as well as educational tools or technologies, have been widely implemented or incorporated in teaching and learning of mathematics. All these aims not only to enhance the teaching and learning but also to help students who have difficulties in mathematics learning.

Agent technology has emerged in information technology (IT) in the 1990s. An agent that is deployed in IT systems could refer as a computer system that autonomous, can adapt to and interact with its environment (Odell, 2010). Agent technology that comes with the feature of intelligence information systems has recently become a popular technology in education too with the intention to promote education or to support learning processes.

In a learning process, the lecturer and the tutor could know the understanding level of a student by reviewing the solution of the given problem solved by the student and interacting (question and answer) through conversation. These types of open-ended human-to-human conversations could definitely provide a great success in teaching and learning process. However, it is not easy 
to practice in a large scale class. The interesting problem is; can this type of interaction between a student and a tutor be replaced by a student and a computer? Human-to-computer conversations are one of the agent technologies that allows human and computer to talk in natural language. This human-to-computer conversation is term as conversation-based learning agent in this study.

There are three phases in this project:

1) To design a proposed framework of conversation-based learning agent and mathematics achievement.

2) To design and to develop a conservation-based learning agent that could interact with the student in natural language,

3) To examine the effect of using the conversation-based learning agent.

The aim of this study is to develop a conversation-based learning agent to help students on learning mathematics and to evaluate the effect of incorporating this conversation agent on students' achievement in mathematics. However, at this point, only the first phase of the project is done and is to be discussed in this paper.

\section{METHODOLOGY}

\section{A. Participants}

This project will be conducted at a private university in Malaysia. The participants in this study are undergraduates who registered for the subject of Mathematics Techniques and Probability. These students age may range from 19 to 30 years old and the sample consists of multiracial such as Malays, Chinese, Indians, and others. There might be international students such as Arabians, Africans, Iranians, Indonesians, Bangladeshis, etc. participated in this study.

\section{B. Instruments}

There are two instruments for this study: an online survey and an achievement test.

The online survey is an instrument that is used to collect students' general information such as age, gender, races, nationality, courses, etc. There is a question to collect information on students' knowledge about the conversation-based learning agent. This survey will be administered before the study. With the general information of students, future analysis such as the relationship between gender and achievement before and after the use of conversation-based learning agent, the relationship between races and achievement before and after the use of conversation-based learning agent, etc. can be conducted.

The achievement test is to be used for collecting data on students' achievement in mathematics. This instrument will be served as pre-test and post-test. In other words, it will be given to students before they use the conversation agent and after they use the conversation-based learning agent for learning mathematics. It is a self-designed test paper consists of five structure mathematics questions. The questions are designed based on the mathematics syllabus and the standard of exam papers in the university in order to ensure the reliability of the instrument. The total score of the test is 100 . The instrument will be validated by a group of experienced mathematics lecturers in the university.

\section{Research Procedure}

First, the proposed plan at the beginning of the first phase will be the designing of the lessons, the preparation of the contents of the conversation-based learning agent, the preparation of the instruments and at the same time the designing of the framework. At the time of writing this paper, the online survey had been conducted and the proposed framework had been designed. In the second phase of this project, the conversation-based learning agent will be designed and developed. The completed conversationbased learning agent system will be exposed to students at the same time as the human lecturer teaching the lesson at which this conversation-based learning agent is used as a blended learning tool. Before the students are exposed to the conversation-based learning agent to study the designed mathematics lesson, they will be given a pre-achievement test. This conversation-based learning agent will serve as a lecturer, a tutor and a mentor where its job is the same as a human lecturer and a human tutor. Students will interact with the conversation-based learning agent through natural language conversation to learn the lesson. After the students have completed the lesson with the conversation-based learning agent, they will be given a post-achievement test. Data collected from pre and post-achievement test will be analyzed using SPSS or other statistical analysis tools. Statistical tests such as descriptive statistics, $t$-test, and etc. will be conducted using 5\% significance level. The effectiveness of using the developed learning agent in students' achievement of the use of conversational-based learning agent in learning mathematics will be evaluated using data collected from the pre and post-achievement test.

\section{PROPOSED FRAMEWORK OF INCORPORATING CONVERSATION-BASED LEARNING AGENTS}




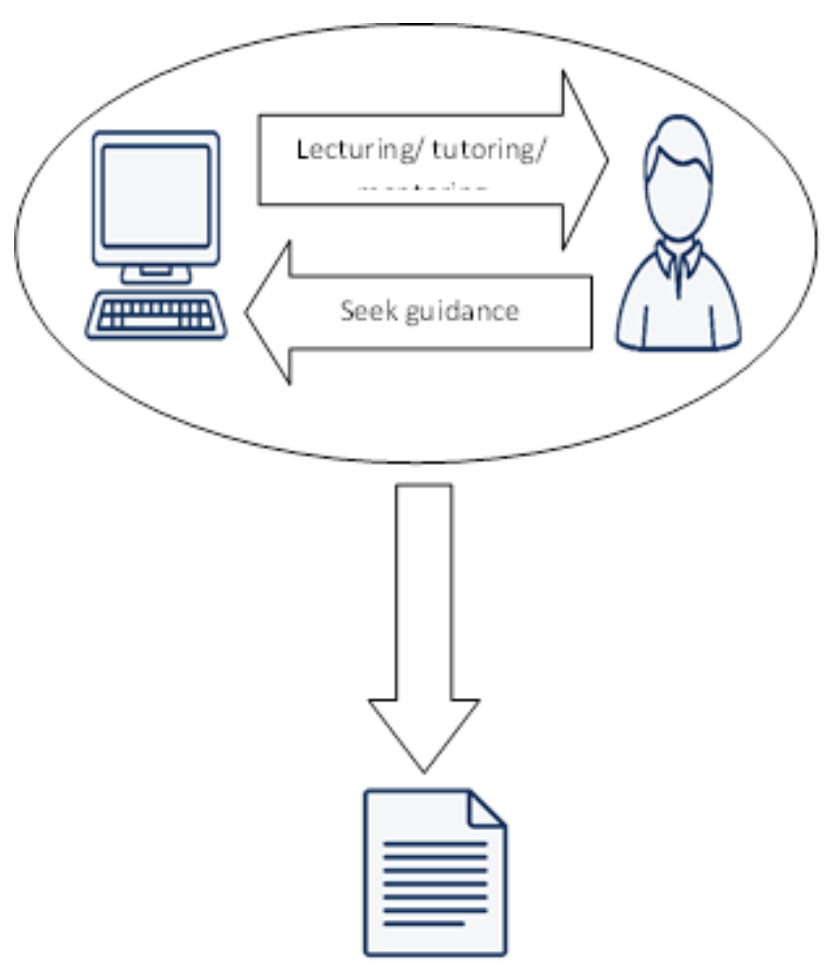

Fig 1: Proposed Framework of Conversation-based Learning Agent

As mentioned earlier, this project aims to incorporate conversation-based learning agents in teaching and learning and to examine students' achievement on the incorporation of conversation-based learning agents in teaching and learning in order to improve their learning and increase their interest in mathematics. Therefore, it is proposed that the conversation-based learning agents are used in teaching and learning. The proposed framework (Fig. 1) was designed.

As we can see from Fig. 1, there is a two-way interaction between the conversation-based learning agents and students. Students will learn mathematics with the agents or seek guidance and help from the agents whenever they encounter problems. Then, the agent will provide helps in the forms of lecturing, tutoring or mentoring to students. With this interaction, students' learning is eased by the agents, and their difficulty in mathematics could be reduced, which in turn improve their mathematics achievement.

\section{SOME FINDINGS OF PHASE 1}

At the time of this paper is produced, only Phase 1 was conducted. The online survey on students' general information such as age, gender, races, nationality, courses, etc. was conducted. Some data on this general information have been analyzed. There were 200 undergraduates at a private university involved in Phase 1, which consisted of 131 males and 69 females. Of them, $25.5 \%$ is Malay, $55.5 \%$ is Chinese, $10.5 \%$ is Indian and $8.5 \%$ is others. Furthermore, 55.5\% of them are aged $19-21$ years old, $36 \%$ are aged $22-24$ years old, $7 \%$ aged $25-27$ years old and $1.5 \%$ aged $28-30$ years old.

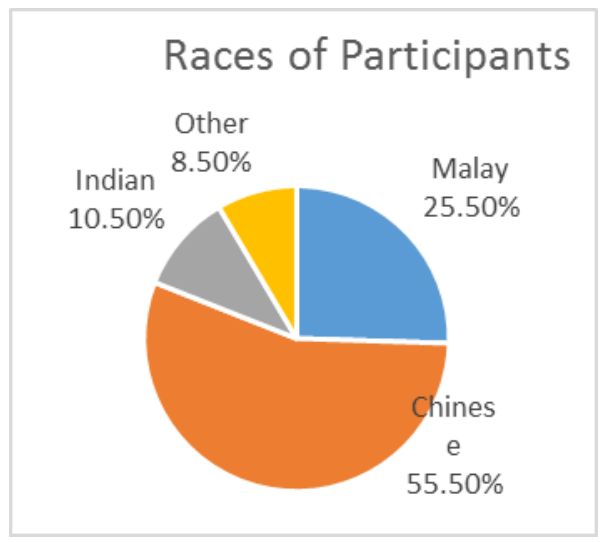

Fig 2: Pie Chart of Races of Participants 


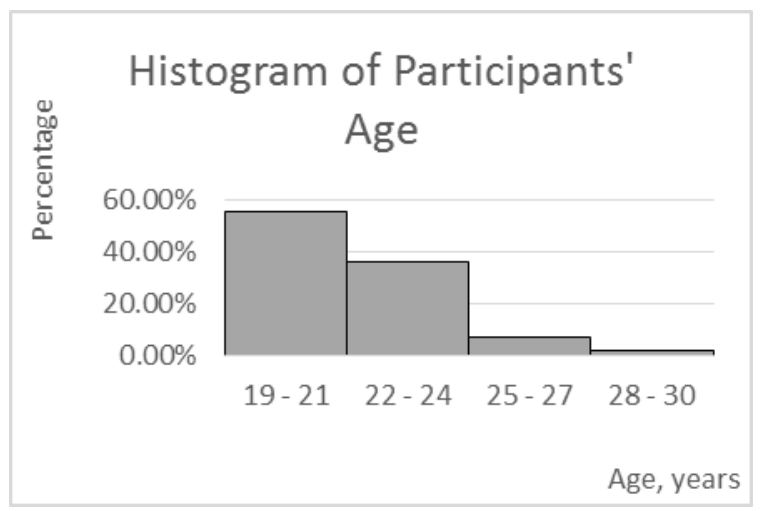

Fig 3: Histogram of Participants’ Age

The results also showed that all students had never heard about the term of conversation-based learning agents, thus all of them had never used the conversation-based learning agents in their learning. Students were also responded that they will use the conversation-based learning agents if they are provided with an opportunity to use it. They are looking forward to use the conversation-based learning agents which they believe that the agents will make the learning fun and interesting.

\section{CONCLUSION}

It is hoped that human-to-computer conversations or term as a conversational-based agent in this study on evaluating the use of conversational-based learning agent may help and ease the teaching and learning process. Besides that, it is also hoped that this study contributes to the design and development of a framework with conversation-based learning agents. The framework will be significant to the instructional designers and educators in helping students to reduce difficulties in learning mathematics and improve performance in mathematics. Eventually, this project may suggest another alternative teaching and learning approach to educators and students.

\section{REFERENCES}

Johnson, D.W., and Johnson, R.T., 1990. Using Cooperative Learning in Math In: Cooperative Learning in Mathematics. Davidson. N. (Ed.), ISBN:0-201-23299-5: 122, Addison-Wesley, 1990.

Ma, X., 2012. Reciprocal Relationships Between Attitude Toward Mathematics and Achievement in Mathematics. The Journal of Education Research, 90(4): 221 - 229.

McCraty, R., Dana, T., Mike, A., Pam, A., and Stephen, J., 2000. Improving Test-Taking Skills and Academic Performance in High School Students Using Heart Math Learning Enhancement Tools. Institute of Heart Math.

Odell, J., 2010. Agent Technology: An Overview. Retrieved from http://www.jamesodell.com/Agent_TechnologyAn_Overview.pdf on 23rd January 2018.

Rösken, B., and Rolka, K., 2007. Integrating Intuition: The Role of Concept Image and Concept Definition For Students' Learning of Integral Calculus. The Montana Mathematics Enthusiast, ISSN 1551-3440, Monograph 3: 181-204.

Zan, R. and Di Martino, P., 2007. Attitude Toward Mathematics: Overcoming the Positive/Negative Dichotomy. The Montana Mathematics Enthusiast, ISSN 1551-3440, Monograph 3: 157-168. 